\title{
7. Bürgerwehr Typ II: Die »Bürgerstreife Weißensee"
}

\subsection{Rahmenbedingungen}

\subsubsection{Feldzugang}

Der Zugang zu Bürgerwehren zweiten Typs ist weitaus schwieriger als zu denen ersten Typs. Während die »Bürgerstreife Harzberg« von der lokalen Politik und der Polizei Anerkennung für ihr Handeln bekommt, stoßen Typ-IIBürgerwehren auf Kritik. Teilweise sind sie Ziel polizeilicher Maßnahmen. Auch die mediale Berichterstattung ist selten positiv. Das führt dazu, dass Bürgerwehren zweiten Typs skeptisch bis ablehnend auf wissenschaftliches Interesse an ihnen reagieren.

Aus diesem Grund gingen die Anfragen nicht an die Bürgerwehren als Einheit, sondern richteten sich direkt an die privaten Facebook-Profile ihrer Mitglieder. Ich habe mir für die Kontaktaufnahme ein Facebook-Profil angelegt, das nicht mit privaten Bildern und Posts gefüllt ist. Die Anfragen sollten unverfänglich und zugänglich klingen. So habe ich am 21. März 2019 Daniel Huber, Mitglied der »Bürgerstreife Weißensee«, via Facebook folgende erste Nachricht geschickt:

»Lieber Daniel Huber, ich bin Studentin und schreibe eine Arbeit über Bürgerstreifen. Mich interessiert, wie Bürger zur Sicherheit beitragen können. Ich habe auf Facebook gesehen, dass Sie sich zusammen mit der Wahlliste »Freie Bürger Weißensee« für eine Bürgerstreife einsetzen. Hätten Sie Lust, mir davon zu erzählen? Das würde mir für mein Projekt sehr helfen. Ich freue mich von Ihnen zu hören. Viele Grüße, Nina«

Seine Antwort kam vier Stunden später und war zunächst sehr ablehnend: 
»Hallo Nina, heutzutage kann man niemanden mehr trauen. Prinzipiell bin ich gerne zu Gespräche bereit solange eine ehrliche Absicht dahinter steht. Ich habe leider mediale schlechte Erfahrungen gemacht. Viele Crüße«

Um sein Vertrauen zu gewinnen, chattete ich eine Woche fast jeden Tag mit ihm. Dann war er schließlich bereit, mich mit auf Streife zu nehmen. Im Laufe des Kommunikationsverlaufs stellte ich klar, dass es sich bei der Arbeit um eine Doktorarbeit handelt, die veröffentlicht werden würde. Wir verabredeten uns für den Abend des 28.03.2019. Am Abend vorher sagte er ab. Wir schrieben eine Weile hin und her. Schließlich sagte er wieder zu. Ich war mir bis zu unserem Treffen am Abend darauf unsicher, ob er nochmals absagen oder ob er einfach nicht auftauchen würde. Aber als ich mit einem Leihwagen aus Leipzig in Weißensee ankomme und auf den Parkplatz fahre, auf dem wir verabredet sind, wartet Daniel Huber schon auf mich.

Es haben sich für den Abend der teilnehmenden Beobachtung neben Daniel Huber noch zwei weitere Mitglieder der Bürgerwehr angekündigt Christoph Zimmermann und Justus Schulz. ${ }^{1}$ Jedoch taucht Christoph Zimmermann letztendlich nicht auf, und so findet die beobachtete Streife nur mit Daniel Huber und Justus Schulz statt.

Daniel Huber ist für seine Verbindungen zur rechten Szene in Sachsen bekannt. Ich habe Angst, er könnte herausfinden, dass ich als Journalistin arbeite und kritisch über Bürgerwehren berichtet habe. Ich weiß im Vorhinein nicht, wie sich die Situation auf Streife entwickeln würde und in welche Situationen ich alleine mit den Männern und ihren Hunden kommen könnte. Diese Angst hat die Beobachtungssituation während der Streife beeinflusst. Ich habe auf das, was die Mitglieder der Bürgerwehr gesagt haben, oft zustimmende Reaktionen gezeigt. Außerdem habe ich oftmals gelacht, um Situationen zu entspannen, und habe mitunter affirmative oder suggestive Fragen gestellt. ${ }^{2}$

Die Analyse der Bürgerwehr in Weißensee beruht auf den transkribierten Mitschnitten der Feldgespräche (etwa drei Stunden) während der teilnehmenden Beobachtung sowie auf der dichten Beschreibung, die im Anschluss an die Beobachtung angefertigt wurde. Die Feldgespräche orientierten sich am Interviewleitfaden.

1 Auch hier handelt es sich zum Schutz der Privatsphäre um Pseudonyme.

2 Ein Beispiel für anerkennende Reaktionen ist die Szene, als Daniel Huber erzählt, die Bürgerwehr bringe die Wirtin einer Bar nachts nach deren Schicht nach Hause. Vgl. Transkript Bürgerstreife Weißensee I, Zeile 302. 


\subsubsection{Sozialstruktur und polizeiliche Kriminalstatistik des Ortes}

Weißensee ist eine Große Kreisstadt in Sachsen. Die CDU-Oberbürgermeisterin ist seit über zehn Jahren im Amt. Bei den Wahlen zum sächsischen Landtag am 01. September 2019 wurde die AfD stärkste Kraft, gefolgt von der CDU. Seit der Wiedervereinigung erlebt Weißensee ein kontinuierliches Abwandern von Menschen und gehört zu den Regionen Deutschlands, in denen die wenigsten Menschen Abitur machen. Dafür hat fast jeder zweite Weißenseer einen Realschulabschluss. Der Anteil von Menschen mit Migrationsgeschichte liegt in Weißensee bei unter zwei Prozent. Bundesweit sind es 19,2 Prozent.

Die Polizeistatistik für den Freistaat Sachsen weist für 2018 einen Rückgang der Straftaten im Vergleich zum Vorjahr auf. Sachsen liegt mit 6831 Straftaten pro 100 o00 Einwohner knapp über dem Bundesdurchschnitt (6710). Im Landkreis, zu dem Weißensee gehört, sind es weit weniger. Mit 42 Prozent sind Diebstähle die häufigsten begangenen Straftaten in Sachsen. Die politisch motivierte Kriminalität wird zu zwei Dritteln von rechts verübt. ${ }^{3}$

\subsubsection{Soziale Position der Gründer}

Daniel Huber ist 42 Jahre alt, er ist in Weißensee geboren und aufgewachsen und betreibt ein Geschäft. Er lebt mit seiner Frau, seinen Kindern und den Schäferhunden in einem Eigentumshaus. ${ }^{4}$ Daniel Huber ist in einem lokalen Verein aktiv, der die Vorläuferdemonstrationen der Pegida-Märsche organisierte und mit der NPD eng verbunden ist. Daniel Huber ist zweiter Vorsitzender des Vereins.

Neben Daniel Huber sind noch einige weitere Mitglieder der Bürgerwehr im Verein engagiert. ${ }^{5}$ Der Verein wird vom Verfassungsschutz beobachtet. Daniel Huber erzählt das mit einem gewissen Stolz ${ }^{6}$ und ergänzt: »also wenn

Vgl. Sächsisches Staatsministerium des Innern (2019): Medieninformation 036/2019. Online unter: https://www.polizei.sachsen.de/de/dokumente/Landesportal/036XPMX PKSX2018.pdf (Zugriff am 2.1.2021). Sowie: Freie Presse Online vom 3.04.2019: https:// www.freiepresse.de/nachrichten/sachsen/weniger-kriminalitaet-bei-mehr-politisch-m otivierten-taten-artikel10484815 (Zugriff am 2.1.2021).

4 Vgl. Transkript Weißensee I, Zeile 644f.

5 Vgl. Transkript Bürgerstreife Weißensee III, Zeile $235 f$.

6 Vgl. ebd, Zeile 224f. 
das nicht so wär, wär's schlimm $\aleph^{7}$ Daniel Huber ist mit seiner extrem rechten politischen Einstellung in Weißensee bekannt. Das stößt bei einigen Bewohnern sowie bei lokaler Politik und Polizei auf Ablehnung. Doch lässt die große Unterstützung im Ort für die AfD auch darauf schließen, dass Daniel Huber bei vielen im Ort Anerkennung für sein Handeln genießt.

\subsubsection{Institutionelle Einbindung der Bürgerwehr}

Die Bürgerwehr in Weißensee ist kaum institutionalisiert. Die örtlichen Sicherheitsbehörden arbeiten nicht mit der Bürgerwehr zusammen. Im Gegenteil: Bereits am Tag, nachdem Daniel Huber auf Facebook die ersten Fotos von der »Bürgerstreife« postete, besuchte ihn ein Mitarbeiter der Kriminalpolizei Chemnitz und klärte ihn über die Gesetzeslage bezüglich der Patrouillen auf. Daniel Huber sollte unterschreiben, dass er über die rechtliche Situation aufgeklärt wurde. ${ }^{8}$ Auch die Bürgermeisterin steht der Bürgerwehr ablehnend gegenüber. ${ }^{9}$ Zwar ist Daniel Huber in der Kommunalpolitik aktiv, das lässt jedoch keineswegs auf eine institutionelle Einbindung schließen. Daniel Huber erklärt, er wolle im Stadtrat sitzen, um die Politik zu kontrollieren, ${ }^{10}$ der die Bürgerwehr Korruption vorwirft. ${ }^{11}$

Auch wenn die Bürgerwehr nicht in offizielle lokale Strukturen eingebunden ist, so ist sie dennoch in (einem Teil) der Bevölkerung verankert. Bei denen, die ihm politisch nahestehen, ist Daniel Huber sehr bekannt und anerkannt, doch bei denjenigen, die sich von rechtem Denken abgrenzen, findet sein Handeln keine Unterstützung.

\footnotetext{
Ebd., Zeile 246f.

Vgl. Transkript Weißensee I, Zeile 1235f, 1291, 1296.

Vgl. Transkript Weißensee II, Zeile 101-122.

Vgl. Transkript Weißensee I, Zeile 897-902, 960-965.

Vgl. Transkript Weißensee IV, Zeile1968-1977.
} 


\title{
7.2 Analyse der "Bürgerstreife Weißensee»
}

\author{
»Es gibt Leute, die uns provozieren, also \\ als Volk, [...] das muss man sich nicht \\ bieten lassen, und wenn die Regierung \\ [...] nichts dagegen tut, dann passen wir \\ halt auf. ${ }^{12}$
}

Die »Bürgerstreife Weißensee« hat sich im Oktober 2018 gegründet. Daniel Huber postete vier Fotos der Streife auf Facebook, auf denen Männer und Frauen mit Hunden zu sehen sind, die für die Kamera posieren. Es ist dunkel auf den Fotos, und sie sind an unterschiedlichen Orten in Weißensee aufgenommen. Im Hintergrund sind eine Mauer, der Busbahnhof, ein Parkplatz und eine Feuerwehrwache zu sehen.

\section{Abbildung 8: Screenshot eines Facebook-Posts von Daniel Huber}

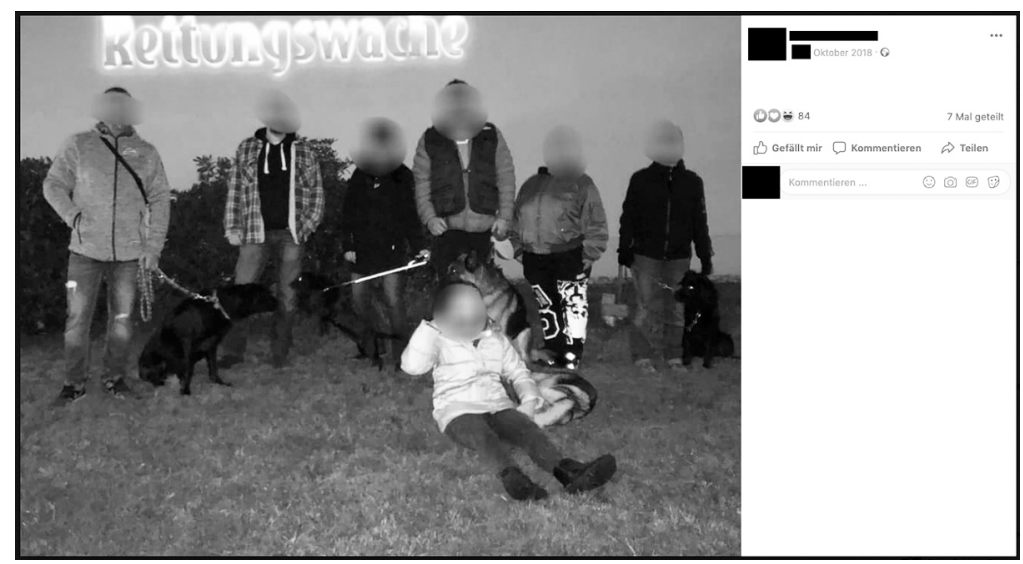

Quelle: Der Screenshot zeigt ein Foto der Bürgerwehr, das vor der Feuerwehrwache aufgenommen wurde. Die Gesichter sind auch auf dem ursprünglichen Post unkenntlich gemacht, die Schwärzungen des Klarnamens von Daniel Huber sind für diese Studie hinzugefügt worden.

Die Mitglieder der Bürgerwehr kannten sich alle bereits bevor sie gemeinsam auf Patrouille gingen. Sie sind befreundet, gemeinsam im Verein tätig 
oder kennen sich von Spaziergängen mit ihren Hunden. Alle Mitglieder der Bürgerstreife haben Hunde, ${ }^{13}$ so lassen sich die ohnehin notwendigen Spaziergänge mit dem Auftreten als Bürgerwehr verbinden. Die Mitglieder verabreden sich mehrmals pro Woche über WhatsApp für ihre Rundgänge als Bürgerwehr. ${ }^{14}$ Die Patrouillen finden immer abends statt und dauern drei bis vier Stunden. ${ }^{15}$

\subsubsection{Legitimationsdiskurse der "Bürgerstreife Weißensee»}

Für die Analyse der »Bürgerstreife Weißensee« werden nun zunächst die Legitimationen diskutiert, die die Bürgerwehr für ihre Patrouillen anführt. Hierfür wird die Kategorie >Legitimation ‘ herangezogen, sie enthält die Codes >Gefahr<, >Fremdeく, >Frauen<, >Polizei< und >Legalität<.

Als Auslöser für die Gründung der Bürgerwehr führen ihre Mitglieder drei mutmaßliche sexuelle Übergriffe auf junge Mädchen in Weißensee innerhalb weniger Wochen im Sommer 2018 an. ${ }^{16}$ Die lokalen Medien berichteten über die Vorkommnisse, und die Polizei rief Zeug*innen auf, sich zu melden.

Mittlerweile hat die Polizei die Verfahren eingestellt. Die Bürgerwehr jedoch ist sich sicher, wer die Täter seien: »die drei stark Pigmentierten in Weißensee. ${ }^{17}$ Damit meinen die Männer die drei in Weißensee untergebrachten unbegleiteten minderjährigen Geflüchteten. ${ }^{18}$ Bereits in der Wortwahl der Bürgerwehr zeigt sich die rassistische Einstellung ihrer Mitglieder. »Stark pigmentiert « ist ein unter Nazis verwendeter Begriff, der benutzt wird, um das N-Wort durch eine andere pejorative Bezeichnung zu umgehen. ${ }^{19}$

In den Augen der Bürgerwehr ist bewiesen, dass die Geflüchteten die Täter waren. Und Daniel Huber hat sofort eine Erklärung parat: Ein Bekannter vom Vater eines der Opfer würde sich mit Funkzellen auskennen und hätte über Handydaten herausgefunden, dass die unbegleiteten minderjährigen

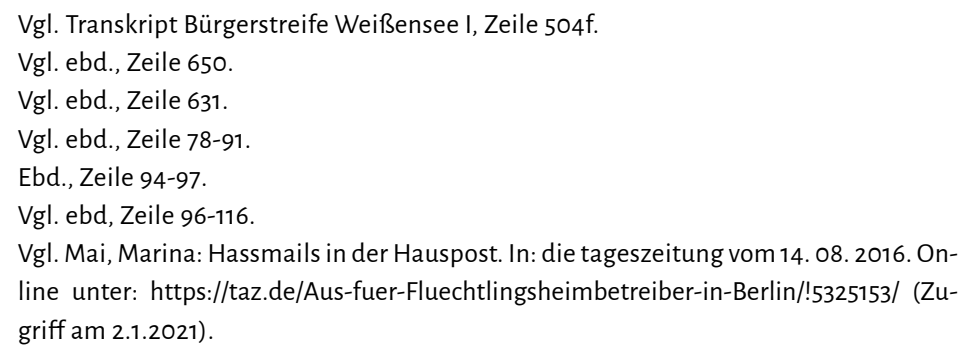
line unter: https://taz.de/Aus-fuer-Fluechtlingsheimbetreiber-in-Berlin/!5325153/ (Zugriff am 2.1.2021). 
Geflüchteten zu den jeweiligen Tatzeiten an den Tatorten waren. ${ }^{20} \mathrm{Mehr} \mathrm{Be}-$ weise als das Wort dieses Mannes existieren nicht. Für die Mitglieder der Bürgerwehr ist das genug.

Daniel Huber interessiert sich sehr für die drei mutmaßlichen Übergriffe. Auch wenn er die mutmaßlichen Opfer kaum persönlich kannte, besucht er sie $\mathrm{zu}$ Hause und versucht mit ihnen über die Vorfälle zu sprechen. ${ }^{21}$ Die Mutter eines der drei Mädchen ist zusammen mit Daniel Huber im Verein engagiert. ${ }^{22}$ Sie erklärt sich bereit, ihre Geschichte zu erzählen und sich dabei von Daniel Huber filmen zu lassen.

Die Mutter erzählt, dass ihre Tochter von »einem Mann südländischen Typs gepackt« wurde, dass er versucht habe, sie »sexuell zu bedrängen«. Er habe ihr "mit der Faust ins Gesicht geschlagen « und sie mit einem Messer am Oberschenkel verletzt. Anschließend fragt sie: »Wie viele sollen noch sterben? Die kommen hier... Junge Männer kommen hier über die Grenze, wo es nicht mal eine Grenze gibt, es wird ja keiner kontrolliert. Die rauben, die morden und dürfen alles. «Auf Facebook ist das Video mit folgendem Text zu finden:

»Zum Thema Sicherheit in Weißensee: Zum persönlichen Gespräch war unser Stadtratskandidat Daniel Huber [...] bei allen drei Überfall-Opfern, welche durch »Schutzsuchende« im Jahr 2018 größere Bekanntheit erlangten. Mit Entsetzen musste man feststellen, dass sich die, welche für die Übergriffe maßgebliche politische Verantwortung tragen, um ihre Verantwortung drücken. Erst durch die Ja-Sager Kultur im Weißenseeer Rathaus (bzw. Stadtrat) war es doch möglich, dass diese Übergriffe überhaupt stattfinden konnten. Ein »NEIN zum Heim« gab es nicht - genauso wenig wie ein Cespräch mit den Opfern. Selbst, als wir unsere (Noch-)Oberbürgermeisterin [...] auf ihre Garantie bzgl. der Sicherheit für die Bürger von Weißensee ansprachen, wollte sie davon nichts mehr wissen.«

Unter dem Text befindet sich ein Link zum Facebook-Post von Daniel Huber, in dem er die erste Patrouille der Bürgerwehr dokumentiert. Daniel Huber versucht, politisches Kapital aus den mutmaßlichen Übergriffen zu schlagen. Die Gründung einer Bürgerwehr ist Teil seiner Strategie.

20 Vgl. Transkript Bürgerstreife Weißensee I, Zeile 134-139.

21 Vgl. Transkript Bürgerstreife Weißensee IV, Zeile 1920-1940.

22 Vgl. Transkript Bürgerstreife Weißensee I, Zeile 1109-1112. 
Zentrale Legitimation der Bürgerwehr für ihr Bestehen ist, die Sicherheit in Weißensee wiederherzustellen, die in den Augen der Bürgerwehr durch die Unterbringung von Geflüchteten im Ort verloren gegangen ist.

»Also sag ich mal, sind wir da, um halt 'n Mindestmaß an Sicherheit zu übergeben. $\ll^{23}$

»Weil die Leute sich ja unsicher fühlen und schon alleine durch die Übergriffe. ${ }^{24}$

Die Bürgerwehr hat sich zur Aufgabe gemacht, Frauen zu beschützen. Denn Männer hätten sich noch keine beschwert, dass es nicht sicher sei für sie in Weißensee, aber Frauen hätten »blanke Angst «. ${ }^{25}$

Das Feindbild ist dabei klar umrissen. Die Ausländer in Weißensee würden »sich >nen Lenz machen und den Frauen nachstellen. ${ }^{26}$ Die Männer von der Bürgerwehr konkretisieren das Feindbild jedoch noch weiter, denn wenn Daniel Huber und Justus Schulz »Ausländer« sagen, so meinen sie damit eine ganz bestimmte Gruppe von Ausländern: Menschen, die aus Afrika kommen oder aus Ländern, in denen der Islam verbreitet ist. ${ }^{27}$ Im Gegensatz dazu beschreiben sie die vietnamesischen Gastarbeiter"innen, die, als es die DDR noch gab, in Weißensee in den Fabriken gearbeitet haben, positiver, jedoch trotzdem rassistisch. Diese hätten »fleißig wie die Bienchen ${ }^{28}$ gearbeitet und sich integriert. ${ }^{29}$

Wenn Justus Schulz die Bürgerwehr legitimiert, kommt er immer wieder auf »die Frauen« zu sprechen, die von der Bürgerwehr vor Ausländern beschützt werden müssten. Wenn Ausländer »den Frauen« nachstellen würden, sei das »ja erstmal vielleicht, wenn das die Frauen wollen, ja ganz gut [...] für die Frauen, wenn sie das brauchen, aber viele wollen's ja nicht. $\ll^{30}$

\footnotetext{
23 Transkript Bürgerstreife Weißensee II, Zeile 156.

24 Transkript Bürgerstreife Weißensee III, Zeile 166.

25 Vgl. Transkript Bürgerstreife Weißensee II, Zeile 547-553.

26 Vgl. Transkript Bürgerstreife Weißensee I, Zeile 593.

27 Vgl. beispielsweise Transkript Bürgerstreife Weißensee IV, Zeile 330-341 sowie Transkript Bürgerstreife Weißensee II, Zeile 499-502.

28 Vgl. ebd., Zeile 473.

29 Vgl. Transkript Bürgerstreife Weißensee IV, Zeile 430-440 sowie Transkript Bürgerstreife Weißensee II, Zeile 476-479.

30 Transkript Bürgerstreife Weißensee I, Zeile 592-595.
} 
Hier zeigt sich, wie abwertend Justus Schulz über Frauen denkt, die mit Menschen aus anderen Ländern denn aus Deutschland zusammen sind. Diese Angst des Weißen Mannes, dass der Schwarze Mann ihm die Frauen wegnimmt, ist ein rassistisches Motiv. ${ }^{31}$ Schwarze Männlichkeit wird in dieser Denkweise als etwas Bedrohliches gesehen, das kontrolliert werden muss. ${ }^{32}$ Dieses rassistische Narrativ zieht sich durch die Argumentationen der Bürgerwehr:

»Die kommen vielleicht aus Kulturen, da sind alle Frauen vermummt und gibt ja Kulturen, musst du Handschuhe anziehen, und sobald die ein Finger sehen, ich will nicht sagen, geht den einer ab, aber, aber die haben dann irgendwie. [...] keine Ahnung, die werden dann erregt oder was und die denken, das ist Freiwild und die Frauen ziehen sich vielleicht so an, dass sie da erobert werden wollen. $\ll^{33}$

Die Gefahr, die die Mitglieder der Bürgerwehr in Weißensee wahrnehmen, ist eng verbunden mit einer Angst vor dem Islam. Der Islam würde allen Männern vorschreiben, »dass man seine Frauen verhüllt. « Die Verwendung des Possesivpronomens deutet darauf hin, dass die Männer der Bürgerwehr ein Vorrecht der deutschen Männer auf deutsche Frauen empfinden. Das Referenzobjekt der von der Bürgerwehr etablierten Bedrohungslage sind also »die deutschen Frauen«. Diese gilt es zu beschützen. Implizit wird dabei ein Anrecht des »deutschen Mannes« auf »seine Frauen« verteidigt. Es handelt sich in dieser Logik bei »deutschen Frauen« somit auch um eine Form von Eigentum, das durch die existenzielle Gefahr der Migration bedroht ist.

Dabei kann die Bürgerwehr bei der Etablierung der Bedrohungslage an ein politisiertes Thema anknüpfen. Das Bild des »übergriffigen Fremden« ist

Vgl. Antonio Amadeu Stiftung (2016): Das Bild des »übergriffigen Fremden«: Warum ist es ein Mythos? S. 4f. Online unter: https://www.amadeu-antonio-stiftung.de/w/files /pdfs/gender_und_rechtsextremismus.pdf (Zugriff am 2.1.2021).

32 Vgl. Eggers, Maureen Maisha (2013): Schwarze feministische Männlichkeitsbilder: Eine paradigmatische Liebe. In: Ballhaus Naunynstrasse (Hg.): Black Lux: Ein Heimatfest aus Schwarzen Perspektiven, 28.08.-30.09.13. Festivalzeitung. Berlin: Selbstverlag. S. 6. Online unter: https://issuu.com/ballhaus-naunynstrasse/docs/black_lux_festiv alzeitung (Zugriff am 2.1.2021).

33 Transkript Bürgerstreife Weißensee IV, Zeile 330-341. 
im medialen Diskurs spätestens seit der Silvesternacht 2015/16 etabliert. ${ }^{34}$ In Weißensee und Umgebung ist das Thema besonders politisiert. Die »Nein zum Heim«-Bewegung und Pegida schürten die Angst vor Kriminalität durch Migrant*innen. Rund um Weißensee waren diese Bewegungen mit am stärksten.

Das referent object in diesem Versicherheitlichungsprozess ist vergeschlechtlicht und sexualisiert. Die Bürgerwehr führt als Legitimation für ihr Bestehen an, Frauen vor sexuellen Übergriffen durch von ihren Mitgliedern als fremd wahrgenommene Menschen schützen $\mathrm{zu}$ wollen. Der Rolle des Beschützers wohnt ein Hierarchieverhältnis gegenüber der zu beschützenden Person oder Personengruppe inne. Die Beschützer sind aktiv, haben Handlungsmacht, treffen Entscheidungen über die Art und Weise, in der Schutz gewährleistet werden soll.

Den Männern der Bürgerwehr ermöglicht diese Rolle, einen Überlegenheitsanspruch auszudrücken und Kontrolle über Frauen auszuüben. Dem Vorhaben, Frauen vor sexuellen Übergriffen schützen zu wollen, liegt die Vorstellung zugrunde, dass die »Ehre« und »Reinheit« der Frauen gesichert werden muss. Letztendlich geht es darum, die alleinige sexuelle Verfügbarkeit der »deutschen Frauen« für die »deutschen Männer« zu sichern.

Dabei hat die von der Bürgerwehr angeführte Bedrohung keinerlei Bezug zu einer materiellen Bedrohungslage. Abgesehen davon, dass sich kein Zusammenhang zwischen Herkunft und Straffälligkeit in Sexualdelikten herstellen lässt, ${ }^{35}$ gibt es in Weißensee gar keine Unterkunft für Geflüchtete. Die wenigen unbegleiteten minderjährigen Geflüchteten, die in einem Haus in Weißensee untergebracht waren, wurden im Herbst 2018 von den Behörden an einem anderen Ort untergebracht. ${ }^{36}$ Diesem Einwand gegen ihre Argumentation kommt die Bürgerwehr zuvor:

»Es ist ja immer so der Kritikpunkt, dass wir gar keine Ausländer hier haben oder Asylanten und äh, dass wir das bloß aus dem Fernsehen kennen, wie schlimm das ist, aber viele von uns sind durchaus weit gereist und kennen die Zustände in Berlin und anderswo, und wir wollen die nicht hier haben, also die Zustände, dass die Kriminalität steigt. [...] Wir haben schon die

34 Vgl. Antonio Amadeu Stiftung (2016): Das Bild des »übergriffigen Fremden«: Warum ist es ein Mythos? S. 3. Online unter: https://www.amadeu-antonio-stiftung.de/w/files/ pdfs/gender_und_rechtsextremismus.pdf (Zugriff am 2.1.2021).

35 Vgl. ebd., S. 3.

36 Vgl. Transkript Bürgerstreife Weißensee I, Zeile 356-362. 
Kontakte, gerade nach Chemnitz und sowas, da geht niemand mehr alleine zur Tramhaltestelle. ${ }^{37}$

Auch wenn vordergründig Frauen beschützt werden sollen, so zeigt sich hin und wieder, dass auch die Männer der Bürgerwehr selber Angst haben. Justus Schulz spricht beispielsweise von »Migranten-Gangs « von denen er sich bedroht fühlt, weil er sich auf einer sprachlichen Ebene nicht mit ihnen verständigen könne. ${ }^{38}$ Meistens jedoch formulieren die Männer die Gefahr nicht in Bezug auf ihre eigene Person, so können sie umgehen, eigene Ängste und somit Schwäche einzugestehen. Stattdessen führen sie eine Gefährdung von Frauen an: Bei der Frage, wie offen die deutsche Gesellschaft ist, hätten »die Frauen mit am meisten $\mathrm{zu}$ verlieren«. Als Mann sollte einem die »MachoKultur« des Islams gefallen: »Frau verhüllen, gut, dann braucht man keine komischen Kleider mehr kaufen. ${ }^{39}$

Die Männer wollen selber keine Opfer sein, denn Opfer-sein stellt ihr Idealbild von Männlichkeit infrage. Wenn sie dennoch Angst empfinden, kanalisieren sie diese, indem sie entweder die Bedrohung zu einer Bedrohung für Frauen erklären oder die Gesamtheit der Deutschen zu Opfern stilisieren, beispielsweise zu Opfern der Politik der Regierung Merkel. ${ }^{40}$ Wenn alle Deutschen Opfer sind, dann passt das Narrativ wieder zur ihrer Identität als Männer, die sich gegen eine angebliche Unterdrückung wehren. Aus diesem Grund beschreibt die Bürgerwehr die Bedrohungslage nur in Bezug auf konkrete Gefahren, wenn diese Frauen betreffen. Andernfalls wird eine diffuse Unsicherheit in Bezug auf Migration als Bedrohung für die »deutsche Identität« formuliert:

»Es gibt Leute, die uns provozieren, also als Volk, möchte ich mal sagen, und das muss man sich nicht bieten lassen, und wenn die Regierung, sprich Stadt, wie auch immer, oder Landkreis da nichts dagegen tut, dann passen wir halt auf. $\ll^{41}$

Die Bürgerwehr etabliert eine Trennung von »wir« und »die anderen«, ein gefährliches Außen bedroht die Gemeinschaft. Es wird eine Notwehrsituation

\footnotetext{
37 Vgl. Transkript Bürgerstreife Weißensee I, Zeile $430 f$.

38 Vgl. Transkript Bürgerstreife Weißensee II, Zeile 417-425.

39 Vgl. Transkript Bürgerstreife Weißensee I, Zeile $820 \mathrm{ff}$.

40 Vgl. ebd., Zeile 998-1007.

41 Ebd., Zeile 582-585.
} 
empfunden, die die Gründung einer Bürgerwehr zum Selbstschutz der Gemeinschaft unvermeidbar erscheinen lässt. Die Notwehrsituation erscheint besonders dramatisch, weil die Gemeinschaft als von den Instanzen, die sie eigentlich schützen sollten, im Stich gelassen beschrieben wird. In den Augen der Bürgerwehr greift die Politik auf nationaler und lokaler Ebene nicht ein oder ruft die Bedrohungslage überhaupt erst hervor, indem sie Migration fördere oder zu wenige Polizeikräfte beschäftige.

Um die Bedrohungslage weiter zu bekräftigen und ihre Patrouillen $\mathrm{zu}$ rechtfertigen, bedient sich die Bürgerwehr in Weißensee also auch dem Narrativ »es gibt zu wenig Polizei«. Das Polizeirevier in Weißensee ist im Zuge von Rationalisierungsmaßnahmen nur noch tagsüber besetzt. Die Bürgerwehr fordert, dass es wieder 24 Stunden besetzt ist. Polizeipräsenz sei notwendig, damit die Leute in Sicherheit und Ruhe schlafen könnten. ${ }^{42}$

Auch wenn die Arbeit der Polizei wertgeschätzt wird ${ }^{43}$ und mehr Polizei im Ort gefordert wird, will die Bürgerwehr nicht mit der Polizei zusammenarbeiten. Es gibt keinerlei Kontakt oder Zusammenarbeit: »Wir sind denen ja mehr ein Dorn im Auge. ${ }^{44}$ Kurz nachdem Daniel Huber die ersten Fotos von der Bürgerwehr auf Facebook veröffentlichte und damit die Gründung der »Bürgerstreife Weißensee « kundtat, bekam er Besuch vom Staatsschutz, ${ }^{45}$ der ihn darüber aufgeklärt habe, was die Bürgerwehr dürfe und was nicht. ${ }^{46}$

Daniel Huber sagt, das sei kein Problem. Er sei mit dem Herrn vom Staatsschutz per Du, der habe nur noch zwei Jahre bis zur Rente und kein Problem mit dem, was Daniel Huber mache. ${ }^{47}$ Daniel Huber stellt die Begegnung dar, als sei sie ein Pflichtbesuch aus dienstlichen Gründen gewesen, er scheint keine Konsequenzen zu befürchten.

Es zeigt sich jedoch auch an mehreren Stellen, dass die Bürgerwehr mit der Arbeit der Polizei nicht zufrieden ist. Die Polizei habe die Suche nach den Tätern der mutmaßlichen sexuellen Übergriffe in Weißensee eingestellt und sei den Hinweisen der Eltern nicht nachgegangen. Es werde alles unter den Teppich gekehrt. ${ }^{48}$ Die Bürgerwehr glaubt zu wissen, wer die Täter sind - die

\footnotetext{
42 Vgl. Transkript Bürgerstreife Weißensee I, Zeile 448-453.

43 Vgl. Transkript Bürgerstreife Weißensee IV, Zeile 565-ff sowie Zeile 578-599.

44 Transkript Bürgerstreife Weißensee I, Zeile $720 \mathrm{ff}$.

45 Vgl. Transkript Bürgerstreife Weißensee IV, Zeile 1235.

46 Vgl. ebd, Zeile 1291 sowie 1296.

47 Vgl. ebd., Zeile 1261-1271 sowie Zeile 1306f.

48 Vgl. Transkript Bürgerstreife Weißensee I, Zeile 1171-1174 sowie Zeile 94.
} 
unbegleiteten minderjährigen Geflüchteten in Weißensee. Ein Schutz der Bevölkerung vor dieser Personengruppe bietet die Legitimationsgrundlage für die Patrouillen der Bürgerwehr. Es ist also auch ein von der Bürgerwehr wahrgenommenes Versagen der Polizei, das ihre Gründung legitimiert.

Die Mitglieder der Bürgerwehr unterstreichen die Notwendigkeit ihres Engagements für die Sicherheit weiter, indem sie auf die Unterstützung ihres Umfelds hinweisen.

»Wir sind, standen vorne, ja, grade wo das Casino ist. Die kamen raus, die Frauen, haben sich bedankt, dass wir da sind. ${ }^{49}$

Unter einem Foto der Bürgerwehr, das Daniel Huber auf Facebook postete, kommentiert eine Userin: »Wier [sic!] Frauen brauchen euch «. Sie ist nicht mit Daniel Huber auf Facebook befreundet. Sie scheint auf die Bürgerwehr aufmerksam geworden zu sein, weil jemand, dem sie auf Facebook folgt, den Beitrag geteilt hat. Die Anerkennung kam also nicht aus Daniel Hubers Bekanntenkreis.

Es gibt auch zwei oder drei Frauen, die aktiv bei der Bürgerwehr mitlaufen..$^{50}$ Diese wollten jedoch für diese Studie nicht zur Verfügung stehen. Auf zwei der Fotos, die Daniel Huber von der Bürgerwehr auf Facebook postet, sieht man jedoch deutlich, dass Frauen dabei sind. Zwei tragen unscheinbare Jacken, die bis über die Hüfte reichen. Eine trägt eine Bomberjacke und eine Jogginghose mit einem Pitbull und einer » 8 « darauf. Auf dem anderen Foto trägt ein vermutlich weibliches Mitglied der Bürgerwehr eine schwarze Sweatshirt-Jacke, eine Bauchtasche und Joggingschuhe. Ihr Kleidungsstil ist klassisch männlich konnotiert und sie ist kräftig gebaut, neben ihr sitzt ein großer Schäferhund. Ihre Haltung auf dem Foto ist nicht von der der Männer zu unterscheiden.

Die Frauen der Bürgerwehr übernehmen einen männlichen Habitus, ${ }^{51}$ um den Anforderungen der Bürgerwehr, physische Stärke zu symbolisieren, genügen zu können. Diese Frauen sind in den Augen der Bürgerwehr keine Opfer, die es zu beschützen gilt, sie schützen sich selbst und sind als Teil der Bürgerwehr auch befähigt, andere Frauen zu schützen.

\footnotetext{
49 Vgl. ebd, Zeile $1193 f$.

50 Vgl. Transkript Bürgerstreife Weißensee I, Zeile 805-810.

51 Vgl. hierzu Bourdieu, Pierre (1997): Die männliche Herrschaft. In: Krais, B./Dölling, I. (Hg.): Ein alltägliches Spiel. Ceschlechterkonstruktionen in der Praxis. Frankfurt a.M.: Suhrkamp. S. 153-217.
} 
Die Bedrohungslage, die durch die existenzielle Gefahr der Migration in Bezug auf die Referenzobjekte »Frauen« und »deutsche Identität« etabliert ist, wird noch durch eine weitere Gefahr ergänzt. Unter einer Brücke in der Nähe des Bahnhofs etablieren die Männer von der Bürgerwehr eine neue Gefahr, die ihre Präsenz im öffentlichen Raum notwendig macht:

»Wir haben ja hier im Grenzgebiet, sag ich mal, zur Tschechei ${ }^{52}$ viel mit Crystal Meth zu tun angeblich, also ich nicht, und aber was man so halt so mitbekommt, (.) soll das wohl echtes Drogenproblem sein (.), und irgendwo muss es ja gekauft und verkauft werden. ${ }^{53}$

Auch hier kann die Bürgerwehr an ein bereits politisiertes Thema anknüpfen. Insbesondere nachdem 2016 bekannt wurde, dass der Grünen-Politiker Volker Beck Crystal Meth nahm, stiegen die Medienberichte über Crystal Meth sprungartig an. Studien über die Wirkung und den Konsum der Droge wurden teilweise falsch zitiert und skandalisiert, ${ }^{54}$ sodass eine weite Verbreitung der Droge in breiten Gesellschaftsschichten suggeriert wurde.

Das Referenzobjekt, das durch die Bedrohung, die von Drogendealern ausgeht, in Gefahr ist, ist einerseits die Gesundheit der Bürger*innen, mehr noch aber »Anstand«, »Recht und Ordnung«. Auch das Feindbild des Drogendealers passt gut mit dem rassistischen Bild des »übergriffigen Fremden« zusammen. So werden in Filmen Drogendealer meistens von Schwarzen dargestellt. ${ }^{55}$

Auch bei der Bedrohung, die von Crystal Meth ausgeht, wird die Kriminalität im Außen verortet, gegen das sich ein bedrohtes Innen verteidigen muss. So schreibt die Welt: »Die Crystal Meth-Flut kommt aus Tschechien« und zeigt eine Übersichtskarte, auf der die Crystal Meth-Funde markiert sind. Die meisten roten Punkte liegen in Sachsen. Zwischen 2009 und 2015 hat sich

52 Die Wortwahl »Tschechei« wird in Tschechien mit der NS-Besatzung ab dem Frühjahr 1939 in Verbindung gebracht. Hitler hatte »von der Zerschlagung der Rest-Tschechei« gesprochen. Vgl. Mai, Marina: Hassmails in der Hauspost. In: die tageszeitung vom 14. 08. 2016, Online unter: https://taz.de/Aus-fuer-Fluechtlingsheimbetreiber-in-Berlin/ !5325153/ (Zugriff am 2.1.2021).

53 Vgl. Transkript Bürgerstreife Weißensee II, Zeile 252-255.

54 Vgl. Wild, Daria (2014): »Die Medien machen Werbung für Crystal Meth«. In watson.ch-Online: https://www.watson.ch/wirtschaft/drogen/386973177-die-medien-mach en-werbung-fuer-crystal-meth (Zugriff am 2.1.2021).

55 Vgl. bspw. Covington, J. (1997): The Social Construction Of the Minority Drug Problem. In: Social Justice, 24(4 (70)), S. 117-147. 
die sichergestellte Menge der Droge in Sachsen verzehnfacht. ${ }^{56}$ Die relative Grenznähe Weißensees wird in diesem Zusammenhang als bedrohlich wahrgenommen.

Zusammenfassend lässt sich also feststellen, dass die Bürgerwehr eine Bedrohungslage etabliert, in der Migration und Drogendealer als Gefahr für die Referenzobjekte deutsche Identität, Eigentum (Frauen) sowie Anstand, Recht und Ordnung dargestellt wird. Dieses Setting verknüpft Ereignisse im sozialen Umfeld gezielt mit politischen Forderungen und ermöglicht die Legitimation einer Bürgerwehr, deren Patrouillen die Sicherheit bezüglich der Referenzobjekte wiederherstellen sollen.

\subsubsection{Motivation der Mitglieder für die Gründung der Bürgerwehr}

Die Bürgerwehr in Weißensee verbindet gezielt Bedrohungen, die aus konkreten Ereignissen im sozialen Nahfeld entstehen, mit politischen Forderungen der Mitglieder der Bürgerwehr, denen sie bereits vor Gründung der Bürgerwehr durch die Teilnahme an Pegida und dem Engagement im Verein Ausdruck verliehen haben. Ihr Auftreten als Bürgerwehr soll einerseits die Sicherheit für die lokale Bevölkerung (wieder-)herstellen, gleichzeitig verleihen die Patrouillen diesen politischen Forderungen Nachdruck und ermöglichen den Mitgliedern eine Stabilisierung fragiler Männlichkeitskonstruktionen. Das Ausdrücken politischer Meinungen mittels der Streifen sowie die Möglichkeit, einen bestimmten Habitus auszuleben, bilden die Motivationen zweiter Ordnung. Sie existieren parallel zum Bedürfnis der Bürgerwehr-Mitglieder, die Sicherheit in Weißensee wiederherzustellen (vgl. Kapitel 7.2.1). Diese Motivationen für die Gründung der Bürgerwehr werden im Folgenden diskutiert. Hierfür werden die Kategorien >Abstiegsangst< und >Habitus` analysiert. Die Kategorie >Abstiegsangst` enthält die Codes >Benachteiligung $<$, >Privilegien $<$ sowie >Staat/System $\prec$. Die Kategorie >Habitus enthält die Codes >Männlichkeit<, >Kompetenzく, >audience und >Hilfsmittel .

Die Mitglieder der Bürgerwehr zeigten in verschiedener Form Ängste vor sozialem und ökonomischem Abstieg. Diese Ängste sind eng verwoben mit dem Gefühl, benachteiligt zu werden. Sie haben Angst vor gesellschaftlichen

56 Vgl. Flade/Hinrichs/Schlesier: Crystal Meth ist die Droge der Generation Selfie. In: Welt Online vom 23.11.2014 https://www.welt.de/politik/deutschland/article134620734/Cryst al-Meth-ist-die-Droge-der-Generation-Selfie.html (Zugriff am 2.1.2021) 
Veränderungen und vor der Zukunft. Das wird beispielsweise deutlich, wenn sie beschreiben, dass zu Zeiten der DDR vieles besser gewesen sei:

»Früher hast du überall, hast du deine Poststation gehabt, da saß 'ne Postmieze, konnste deine Briefe aufgeben und so weiter, hat's genauso, dass an de Bahnhöfe war ja auch Sicherheit, da hattste jemand dasitzen der konnte nach'm Rechten gucken, das haben sie alles, also großteils, abgeschafft, so, aber was dann auf jeden Fall auch Kritikpunkt ist, wo, wo bleiben denn die Gelder, was wird mit unseren Steuern gemacht, wird das alles jetzt nach Griechenland verfrachtet? «77

Ein diffuses Gefühl, zu kurz zu kommen, wird dabei immer wieder in Form von Verteilungskämpfen geäußert, bei denen sich die Mitglieder der Bürgerwehr von der Politik ungerecht behandelt fühlen. So war der Auslöser für Daniel Huber, sich politisch zu engagieren, dass seinen Töchtern nicht mehr das Busticket zur Schule bezahlt wurde. Es regt ihn auf, dass dafür kein Geld da sei, und fügt hinzu: »Im Zuge der Flüchtlingskrise, wenn man das so nennen möchte, es sind ja dann auch Gelder aufgetaucht, also es kostet ja alles Geld. ${ }^{58}$

Die Argumentationen driften dabei immer wieder in Verschwörungstheorien ab. Zum Beispiel, wenn die Männer eine "Asylindustrie« beschreiben, die sich in Deutschland etabliert habe und bei der eine Menge Leute mit Geflüchteten Geld verdienen würden. ${ }^{59}$ Oder wenn sie sich darüber aufregen, dass Geflüchtete zwanzig oder dreißig Autos und Luxuslimousinen hätten, ${ }^{60}$ dass sie mit dem Taxi herumfahren und der deutsche Staat dafür bezahlen würde. ${ }^{61}$ Demgegenüber werden Deutsche als benachteiligt dargestellt, ihnen würde von Ausländern die Arbeit weggenommen, ${ }^{62}$ sie würden in die Armut gedrängt, zum Flaschensammeln gezwungen. ${ }^{63}$ Justus Schulz hat zudem Angst, dass die Zinspolitik der Regierung ihm sein Erspartes wegnehmen würde. ${ }^{64}$

\footnotetext{
57 Transkript Bürgerstreife Weißensee IV, Zeile 732-737.

58 Ebd., Zeile 747-790.

59 Vgl. Transkript Bürgerstreife Weißensee II, Zeile $521 \mathrm{ff}$.

60 Vgl. Transkript Bürgerstreife Weißensee IV, Zeile 860ff.

61 Vgl. Transkript Bürgerstreife Weißensee II, Zeile 333-351.

62 »In Nürnberg da gab's in 'ner ganzen Ladenstraße kein deutsches Ceschäft mehr.« Vgl. Transkript Bürgerstreife Weißensee I, Zeile 831-836.

63 Vgl. Transkript Bürgerstreife Weißensee II, Zeile 682-688.

64 Vgl. ebd., Zeile 618-630.
} 
Diese Opfermentalität ermöglicht es den Männern von der Bürgerwehr, Formen des Ausschlusses zu verarbeiten, die sie nicht benenn können sowie persönliches Scheitern zu erklären, das eigentlich nicht mit einem Bild von Männlichkeit vereinbar ist, das als zentrale Attribute Souveränität, Überlegenheit und Autonomie besitzt. Wenn alle Deutschen Opfer sind, dann sind sie unverschuldet und nicht alleine in dieser Position der Schwäche. Auf diese Weise können sie ihre fragile und spannungsreiche Männlichkeitsidentität stabilisieren.

Diese Angst vor sozialem und ökonomischem Abstieg ist auch eine Angst vor dem Verlust von Privilegien, die die Mitglieder der Bürgerwehr als Weiße deutsche Männer haben. Durch die Migrationsbewegungen seit 2015 wurden globale Ungleichheiten in Deutschland direkter sichtbar. Das führt zu einer Verunsicherung, welche Auswirkungen diese Veränderungen auf die Vorrechte haben, die Menschen mit einem deutschen Pass in der Welt genießen. Die Männer von der Bürgerwehr zeigen hier eine eindeutige Angst davor, ihre Privilegien als Deutsche zu verlieren:

»Das funktioniert nicht, das wird auch auf Dauer nicht gut gehen ne, da brauch ich kein Prophet zu sein, das geht schon rein rechnerisch gar nicht, entweder bezahlen wir dann alle fünfzig Prozent Krankenkassenbeiträge. $\ll^{65}$

»Wir sind hier in System angekommen, was auf Lug und Betrug aufbaut und wo man sich dann fragt, zum Beispiel sind die Renten sicher. ${ }^{66}$

Hinzu kommt eine allgemeine Angst vor dem Islam: »dass die halt Europa durch die Bäuche ihrer Frauen erobern wollen. " ${ }^{67}$ Sowie eine Angst davor, dass es »Chaos « geben könnte: »Ich will da wirklich nicht drüber nachdenken, was hier wird in zehn Jahren. ${ }^{68}$

»Das wird dann im Bürgerkrieg enden zwangsweise, also diese Kriegsgefahr, so wie du's jetzt in der Ukraine siehst, kann das bei uns auch losgehen. « ${ }^{69}$

65 Transkript Bürgerstreife Weißensee II, Zeile 567ff.

66 Ebd., Zeile 630ff.

67 Transkript Bürgerstreife Weißensee IV, Zeile $1762 f$.

68 Vgl. Transkript Bürgerstreife Weißensee I, Zeile 639f.

69 Transkript Bürgerstreife Weißensee II, Zeile 574f. 
Diese Ängste werden von der Bürgerwehr mit einer allgemeinen Systemkritik verbunden. Die Bürgerwehr lehnt die parlamentarische Demokratie, wie sie in Deutschland gegenwärtig umgesetzt wird, ab. Die Parteien würden sich nicht voneinander unterscheiden und ihre Versprechungen nicht halten. ${ }^{70}$ Diese »Politikunzufriedenheit ${ }^{71}$ äußern beide Mitglieder der Bürgerwehr in verschiedenen Zusammenhängen. Die Kritik wird mit Verschwörungstheorien vermischt, es gäbe Korruption, ${ }^{72}$ man werde vom "Staatsfunk belogen " $^{73}$ und Deutschland entwickele sich zu einer »DDR 2.0«. ${ }^{74}$ Merkel und Seehofer gehörten verhaftet. ${ }^{75}$

»In meinen Augen ist das Faschismus was in Berlin sitzt, was dort vor sich geht ne, es wird ja am Volk vorbeiregiert, das interessiert die nicht. ${ }^{76}$

Daniel Huber hat eine »Wut auf dieses ganze kranke System (.). Es stinkt nun an jeder Ecke zum Himmel und die tun so, als ob das Friede, Freude, Eierkuchen wär. «77 Er nahm regelmäßig an Pegida-Demonstrationen teil und wurde wegen eines Plakates, das zu Gewalt gegen Personen aufrief, zu einer Geldstrafe verurteilt.

Ergänzt wird das Narrativ, dass die Politik sie ungerecht behandele, durch das Narrativ, dass der Westen Ostdeutschland bei der Wiedervereinigung unfair behandelt habe. Die Mitglieder der Bürgerwehr haben das Gefühl, als Ostdeutsche bei den Treuhandverhandlungen über den Tisch gezogen worden zu $\operatorname{sein}^{78}$

Einige Mitglieder der »Bürgerstreife Weißensee« sympathisieren mit den Reichsbürgern. Justus Schulz und Daniel Huber kennen die Theorien der Reichsbürger ${ }^{79}$, und auch, wenn sie sich selbst nicht so bezeichnen würden, keinen »gelben Schein ${ }^{80}$ hätten, findet Justus Schulz, die Reichsbürger hät-

\footnotetext{
70 Vgl. Transkript Bürgerstreife Weißensee I, Zeile 905-913.

71 Ebd., Zeile 922.

72 Vgl. Ebd., Zeile 937 sowie Transkript Bürgerstreife Weißensee IV, Zeile 1957.

73 Transkript Bürgerstreife Weißensee I, Zeile 922.

74 Ebd., Zeile 931.

75 Vgl. Transkript Bürgerstreife Weißensee IV, Zeile 1315-1318.

76 Transkript Bürgerstreife Weißensee I, Zeile $1005 \mathrm{ff}$.

77 Transkript Bürgerstreife Weißensee IV, Zeile $899 \mathrm{ff}$.

78 Vgl. Transkript Bürgerstreife Weißensee II, Zeile 438-465.

79 Vgl. ebd., Zeile 710-805.

80 Transkript Bürgerstreife Weißensee VI, Zeile 82.

Mit dem »gelben Schein« meinen Reichsbürger den Staatsangehörigkeitsausweis.
} 
ten ein "paar konkrete Punkte, die mal geklärt werden sollten. ${ }^{81}$ Außerdem gäbe es noch einen »Spezialisten « für das Thema bei der Bürgerwehr. ${ }^{82}$

Aber auch auf lokaler Ebene misstraut die Bürgerwehr dem Staat und seinen Institutionen. Die Bürgermeisterin würde die Mitglieder der Bürgerwehr als rechts bezeichnen und nicht mit ihnen reden. ${ }^{83}$ Im Rathaus gäbe es Korruption bei der Vergabe von Bauaufträgen ${ }^{84}$ und die örtliche Polizei würde bei den mutmaßlichen sexuellen Übergriffen in Weißensee nicht richtig ermitteln und Dinge »untern Teppich kehren $« .{ }^{85}$

Neben einer Angst vor sozialem und ökonomischem Abstieg und dem Verlust von Privilegien existiert eine zweite, persönliche Motivation für die Patrouillen: die Stabilisierung einer fragilen und spannungsreichen männlichen Identität. Die Mitglieder performen eine marginalisierte Männlichkeit. Das heißt, sie sind in ihrer Position innerhalb der hierarchischen Binnenschichtung von Männern in einer minderprivilegierten Rolle und erfahren Diskriminierungen von anderen Männern, insbesondere von denjenigen, die Zugang zu einer hegemonialen Männlichkeit haben. Das Auftreten als Bürgerwehr stellt einen Versuch dar, diese Unterdrückungserfahrungen mit internalisierten Anforderungen an Männlichkeit, wie Souveränität und Hegemonie, zu vereinen.

Dieses Bedürfnis nach Stabilisierung ihrer Männlichkeitsidentität zeigt sich bereits in der Art und Weise, wie die Männer der Bürgerwehr die Bedrohungslage etablieren. Sie rechtfertigen ihre Streifen mit dem Schutz von Frauen vor Übergriffen, die angeblich von Geflüchteten aus arabischen und afrikanischen Ländern drohten. Dabei werden diese antizipierten Übergriffe auf Frauen von den Männern der Bürgerwehr als persönliche Provokation empfunden. ${ }^{86}$

Ein deutscher Pass oder Personalausweis reicht ihnen nicht als Nachweis der deutschen Staatsangehörigkeit. Vgl. Homepage des Bundesministerium des Inneren: https://www.bmi.bund.de/SharedDocs/topthemen/DE/topthema-reichsbuerger/to pthema-reichsbuerger.html (Zugriff am 2.1.2021)

81 Transkript Bürgerstreife Weißensee II, Zeile 711f.

82 Vgl. Transkript Bürgerstreife Weißensee I, Zeile 724.

83 Vgl. Transkript Bürgerstreife Weißensee II, Zeile 101-122.

84 Vgl. Transkript Bürgerstreife Weißensee IV, Zeile 1957-1981.

85 Transkript Bürgerstreife Weißensee I, Zeile 1174.

86 Vgl. Transkript Bürgerstreife Weißensee I, Zeile 589-595. 
So können die Männer als Beschützer auftreten und dafür Anerkennung von Frauen bekommen, die sie beschützen. »Wier [sic!] Frauen brauchen euch «, ist in den Kommentaren unter einem Foto der Bürgerwehr auf Facebook zu lesen. Der Habitus des männlichen Beschützers lässt sich auch im Auftreten der Bürgerwehr im öffentlichen Raum beobachten. Daniel Huber strahlt mit seiner Körperstatur bereits Stärke aus. Das bekräftigt er auch direkt, indem er auf seine Körpergröße verweist: »eins-sechsundachtzig gibt schon 'ne gewisse Sicherheit «. ${ }^{87}$

Sein selbstbewusster aufrechter Gang suggeriert physische Stärke, und sein Schäferhund verleiht ihm Autorität. Hunde waren, bevor sie Haustiere wurden, ein Mittel zur Selbstverteidigung. Daniel Huber führt seinen Schäferhund an der Leine, er ist ruhig und gehorsam. Dennoch ist er eine potenzielle Waffe, die Daniel einsetzen kann, wenn er will:

»Das überträgt sich halt, wenn man, wie sagt man, wenn man beruhigt und ausgeglichen ist, das wird, gibt's halt dem Hund wieder mit, ne, wenn jetzt Stress ist, das spürt der ja sofort, mit der, die haben ja nun ihre Instinkte ${ }^{88}$

Obwohl auch die Männer Ängste haben, erscheinen sie nicht direkt als diejenigen, die Angst haben, ${ }^{89}$ es wird eine Bedrohung der Frauen beschrieben, die eine Bürgerwehr rechtfertigt. So müssen die Männer keine eigene Schwäche eingestehen. Auch in den Unterhaltungen der Männer während und nach der Streife zeigt sich, dass die Männer gerne ein Idealbild von hegemonialer Männlichkeit verkörpern würden; eine Form von Männlichkeit die vermeintlich Wissen und Kompetenz besitzt und keine Ängste kennt. Die Unterhaltungen basieren nicht auf gegenseitiger Empathie, es wird nicht auf den anderen eingegangen, das Gegenüber dient lediglich als Publikum, damit das eigene Ego die Bühne erklimmen kann. ${ }^{90}$

\subsubsection{Auswirkungen der "Bürgerstreife Weißensee» auf das lokale Umfeld}

Die Männer der Bürgerwehr verändern durch ihre Patrouillen den öffentlichen Raum, indem sie Präsenz zeigen. Diese Auswirkungen werden im Fol-

\footnotetext{
87 Ebd., Zeile 315.

88 Transkript Bürgerstreife Weißensee III, Zeile $482 \mathrm{ff}$.

89 Vgl. Transkript Bürgerstreife Weißensee II, Zeile $547 f$.

90 Vgl. Dichte Beschreibung Weißensee, S. $8 \mathrm{f}$.
} 
genden diskutiert. Dafür wird die Kategorie spolitische Positionen siert, diese enthält die Codes >Rassismus`, >Macht und `Gewalt‘. Die Gefahr, die die Männer anführen, um ihr Auftreten als Bürgerwehr zu legitimieren, gehe von Geflüchteten beziehungsweise Ausländern aus. Wenn die Männer von Unsicherheit sprechen, kommen sie meistens auch auf Menschen nichtdeutscher Herkunft zu sprechen. ${ }^{91}$ Die Männer nennen sie entweder »Ausländer ${ }^{92}{ }^{92}{ }$ UMAs ${ }^{93}$ (Ablürzung für »unbegleitete minderjährige Ausländer «), ${ }^{94}$ »Nafris ${ }^{95}$ oder Afrikaner $« .{ }^{96}$ Die Bedrohungslage ist in der Wahrnehmung der Bürgerwehr eine »importierte $« .{ }^{97}$

Das zeigt sich auch in der Wahrnehmung, die die Bürgerwehr vom öffentlichen Raum hat. Der pittoreske Marktplatz von Weißensee mit seinen Fachwerkhäusern, dem sauberen Kopfsteinpflaster und den adrett angeordneten Blumenkübeln wird von der Bürgerwehr als Brennpunkt wahrgenommen. ${ }^{98}$ Der Grund: Hier würden sich im Sommer die in Weißensee untergebrachten Geflüchteten aufhalten.

»Im Sommer schon, weil die sitzen dann hier, hier ist kostenlos WLAN und da sitzen auf der Bank, auch die Kinder meistens immer. Wenn die uns sehen, hören, riechen, sind sie verschwunden, hast du auch im Kaufland. (lacht) Na, was du da manchmal gesehen hast, wie die gerannt sind, also wir haben wirklich niemandem getan, auch niemandem angesprochen oder sowas, einfach nur dort stehen und gucken, was machen die. $\ll^{99}$

»Wir zeigen halt körperliche Präsenz «, ${ }^{100}$ sagt Daniel Huber. Zusammen mit den anderen Mitgliedern der Bürgerwehr und den Hunden übt diese physische Präsenz im öffentlichen Raum Macht aus. Menschen, von denen in den Augen der Bürgerwehr eine Gefahr ausgeht, fühlen sich davon bedroht. Die Mitglieder der Bürgerwehr haben ein rassistisches Weltbild, das dazu

Vgl. Transkript Bürgerstreife Weißensee I, Zeile 320-335, insbes. Zeile 331: »mit Ausländern kriegste Kriminalität« sowie Zeile 433-440.

93 Ebd., Zeile 410.

94 Ebd., Zeile 104-129.

95 Ebd., Zeile 684.

96 Transkript Bürgerstreife Weißensee IV, Zeile $325 \mathrm{f}$.

97 Vgl. Transkript Bürgerstreife Weißensee I, Zeile 742.

98 VgI. Transkript Bürgerstreife Weißensee III, Zeile249-254.

99 Ebd., Zeile 253-258.

100 Ebd., Zeile 211. 
führt, dass nur Menschen mit nicht-weißer Hautfarbe diese Machtausübung zu spüren bekommen. In Weißensee gab es Übergriffe auf Geflüchtete, und Daniel Huber ist aufgrund seiner politischen Einstellungen im Ort bekannt. $\mathrm{Da}$ ist es nicht überraschend, dass Menschen, die in das Feindbild der Bürgerwehr passen, eine Konfrontation zu vermeiden versuchen und den öffentlichen Raum verlassen, wenn die Bürgerwehr auf Streife ist. »Die laufen manchmal ganz schnell weg «, ${ }^{101}$ beschreibt die Bürgerwehr die Reaktion von ausländischen Menschen auf ihr Erscheinen. Dieser passiven Machtausübung wohnt ein Gewaltpotenzial inne. Als die beiden Männer sich darüber unterhalten, was man tun müsste, damit »Wirtschaftsflüchtlinge« gar nicht erst nach Deutschland kämen, scherzt Daniel Huber, man könne auch eine Atombombe auf Afrika werfen. ${ }^{102}$

Nach eigenen Angaben hat die Bürgerwehr keinerlei Waffen dabei. Aber die Männer kritisieren diesbezüglich die Rechtslage in Deutschland.

»Man muss mal auch ganz klar sagen, dass im Cegensatz zu anderen Ländern, den Deutschen ja so was wie Selbstverteidigung so oder so mehr oder weniger verboten ist, grad mit Waffenbesitz und so. ${ }^{103}$

Und wenn sie eine Straftat beobachten, würden die Männer von der Bürgerwehr eingreifen: ${ }^{104} »$ Das Überlegen machste dann hinterher, das sind halt Sachen, Reflexe, die da rauskommen. ${ }^{105}$

Im Anschluss an die Patrouille werden beim Bier Geschichten ausgepackt, bei denen potenzielle Gewaltanwendungen deutlich werden. Ein Bekannter habe einen Syrer mit seinen zwei Rottweilern bedroht, angeblich, weil dieser seine Tochter nach Sex gefragt habe. ${ }^{106}$ Daniel Huber selbst habe den Vater eines der mutmaßlichen Opfer sexueller Nötigung aus Weißensee überredet, das Recht nicht selbst in die Hand zu nehmen:

»Bleib zu Hause, das bringt nichts ne. Du machst dich dein Leben, du sitzt zwanzig Jahre im Knast, weil er den [...] abgemurkselt hat. $\ll^{107}$

\footnotetext{
101 Transkript Bürgerstreife Weißensee IV, Zeile 313.

102 Vgl. Transkript Bürgerstreife Weißensee II, Zeile 505.

103 Transkript Bürgerstreife Weißensee III, Zeile 293ff.

104 Vgl. Transkript Bürgerstreife Weißensee I, Zeile 1179.

105 Ebd., Zeile 1183f.

106 Vgl. Transkript Bürgerstreife Weißensee IV, Zeile 1838-1848.

107 Ebd., Zeile 1855-1860.
} 
Hier wird von einem möglichen Mord aus Rache gesprochen. Ähnlich wie bei den Lynchmorden des 20. Jahrhunderts an Schwarzen Menschen in den USA ist hier das Narrativ des »übergriffigen Fremden« in der lokalen Bevölkerung verankert und wird herangezogen, um rassistische Gewaltverbrechen zu rechtfertigen.

Eine Szene während der teilnehmenden Beobachtung ${ }^{108}$ veranschaulicht diese Parallele weiter. Im Anschluss an die Streife sitzt die Bürgerwehr in einer Kneipe und trinkt Bier.

Daniel Huber wechselt plötzlich das Thema, wendet sich mir zu und erzählt, dass er am Tag nach der Cründung der Bürgerwehr Besuch vom Verfassungsschutz bekommen habe. Er holt sein Handy heraus und wischt durch seine Fotos, um das Foto von dem Brief zu suchen, den er dabei ausgehändigt bekam.

Daniel Huber wischt über sein Smartphone, er hält es dabei so, dass ich die Fotos sehen kann. Zwischen Fotos von seinen Kindern und seinem Hund befindet sich ein Foto von Schaltknüppeln in einem Auto. Die vier Cänge sind so in das Metall eingelassen, dass die Form eines Hakenkreuzes zu sehen ist. Daniel Huber bleibt bei diesem Foto kurz hängen, wischt dann ein paar Mal vor und zurück, als wolle er sichergehen, dass ich das Foto auch wirklich gesehen habe. [...]

Noch immer hat Daniel Huber das Foto des Briefs nicht gefunden. Stattdessen zeigt er ein Foto, auf dem eine Puppe mit schwarzer Hautfarbe mit einem Strick am Hals an einer Brücke aufgehängt ist. Er zeigt es erst mir, dann in die Runde. Das ist in Weißensee erklärt er, und er wisse, wer das gewesen sei. Die drei lachen. Daniel Huber benutzt das N-Wort, um die Puppe zu beschreiben. ${ }^{109}$

Die Schwarze Puppe lässt sich als Aufruf zur Lynchjustiz deuten, der nur wenige Monate vor Daniel Hubers Gründung der Bürgerwehr geschah. ${ }^{110}$ Daniel Huber sagt, er wisse, wer das gewesen sei. ${ }^{111}$ Die Schwarze Puppe könnte zudem eine Anspielung auf den Nationalsozialistischen Untergrund (NSU) sein. Uwe Böhnhard hängte 1996 bei Jena eine Puppe an einem Strick an ei-

108 Vgl. Dichte Beschreibung Weißensee, S. $10 f$.

109 Vgl. Transkript Weißensee IV, Zeile 1479 und 1493.

110 Vgl. Dichte Beschreibung Weißensee, S. 11.

111 Vgl. Transkript Weißensee IV, Zeile 1489 und 1499-1505. 
ner Autobahnbrücke auf, die mit einem Davidstern und der Aufschrift »Jude« gekennzeichnet war. ${ }^{112}$

Das Gewaltpotenzial der Bürgerwehr und die explizit antidemokratische Grundhaltung lässt es widersprüchlich erscheinen, die Bürgerwehr als Prozess der Versicherheitlichung zu erfassen. Jedoch beschreibt die Kopenhagener Schule Sicherheit explizit nicht als positives Gut. Vielmehr ist sie das Ergebnis eines sozialen Prozesses, den es empirisch zu beschreiben gilt. Dieser Versicherheitlichungsprozess ist erfolgt, wenn die Gründer aus einer position of authority heraus erfolgreich eine Bedrohungslage (existential threat) beschreiben konnten, die die Maßnahme (extraordinary measure) der Gründung einer Bürgerwehr gegenüber der lokalen Bevölkerung (audience) rechtfertigt.

Die audience akzeptiert den Sprechakt, der in Weißensee eine Bedrohungslage verortet. Das heißt jedoch nicht, dass alle Menschen, die in Weißensee leben, die Ansichten der Bürgerwehr teilen. Doch bei der Europawahl im Mai 2019 sowie bei den Landtagswahlen im September 2019 wurde wie oben erwähnt die AfD stärkste Kraft, dahinter lag die CDU. Und auch auf zivilgesellschaftlicher Ebene sind rechte und rechtsextreme Bewegungen und Organisationen stark verankert. Die öffentliche Debatte um Migration und Sicherheit in Weißensee hat sich also bereits so weit nach rechts verschoben, dass die Argumentation der Bürgerwehr auf Interesse bis Zustimmung stößt. Dabei haben die Gründer der Bürgerwehr eine sehr ambivalente position of authority innerhalb der lokalen Bevölkerung. So hat sich beispielsweise die Bürgermeisterin von Weißensee wiederholt explizit gegen Daniel Huber und seine politischen Aktivitäten gestellt. Dennoch stellt sich in der lokalen Bevölkerung niemand der Bürgerwehr in den Weg, niemand verteidigt den öffentlichen Raum. Die schweigende Mehrheit stimmt zu und akzeptiert die Bürgerwehr in den Straßen ihrer Stadt.

Das Gewaltpotenzial der »Bürgerstreife Weißensee« zeigt die Ambivalenz von Versicherheitlichungsprozessen besonders anschaulich. Die Gründung der Bürgerstreife Weißensee stellt eine Versicherheitlichung in Weißensee dar, obwohl der Ort für einige Menschen durch den Prozess wesentlich unsicherer wird - für diejenigen, die von der Bürgerwehr als Gefahr beschrieben werden, für die Geflüchteten und nicht-Weißen Menschen im Ort.

112 Siehe Ramelsberger, Annette/Schultz, Tanjev: Dokumentation des NSU-Prozesses für Süddeutsche Zeitung-Online: https://gfx.sueddeutsche.de/politik/2016-04-25_nsu-pro zess/article2/index.html (Zugriff am 2.1.2021) 